\title{
Bone Marrow Findings in Patients With Ewing Sarcoma/Primitive Neuroectodermal Tumor
}

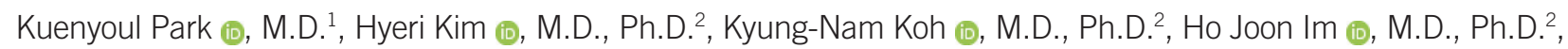

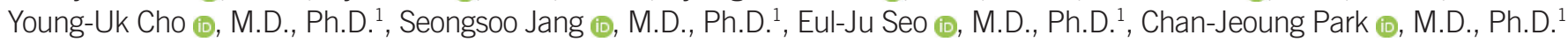
Departments of ${ }^{1}$ Laboratory Medicine and ${ }^{2}$ Pediatrics, University of Ulsan College of Medicine and Asan Medical Center, Seoul, Korea

\section{Dear Editor,}

Ewing sarcoma is the second most common type of bone sarcoma in pediatric patients. The microscopic morphology of Ewing sarcoma family tumor (ESFT) shows small, uniform, round cells with scanty Periodic acid-Schiff (PAS)-positive cytoplasm [1]. Strong, diffuse membranous CD99 expression, a hallmark of ESFT, is observed in $95 \%-100 \%$ of ESFT cases [2].

Bone marrow (BM) metastasis is a poor prognostic factor as compared with other bone metastases [3]. An inexperienced hematopathologist may misdiagnose BM metastasis in patients with ESFT [4]. Various efforts to increase the sensitivity of BM aspiration and biopsy (BMAB), including novel immunohistochemical (IHC) markers such as PAX7, have been reported [5]; however, reports on BM studies of patients with ESFT are limited. We investigated the morphological and IHC characteristics of BM findings in ESFT with BM metastasis.

This retrospective study included a total of 55 patients who were newly diagnosed as having ESFT and who underwent BM studies at Asan Medical Center, Seoul, Korea, between January 2000 and September 2018. This study was approved by the Institutional Review Board of Asan Medical Center (S2020-03140001), which waived the requirement for written informed consent.

We reviewed electronic medical records of all the enrolled pa- tients for clinical synopsis and the first BM findings. BM biopsy specimens or clot sections were stained with anti-CD56 (123C3; Zymed, San Francisco, CA, USA) and anti-CD99 (ERP3097Y; Cell Marque Corp., Hot Springs, AR, USA) using an automated IHC staining system (Ventana Benchmark XT; Ventana Medical Systems, Tucson, AZ, USA).

We applied Pearson's chi-square test and two-tailed Student's t-test for statistical analysis using MedCalc (version 19.2; MedCalc Software Ltd, Ostend, Belgium).

We observed that age, sex, and primary tumor sites were not significantly associated with metastasis at diagnosis. Primary tumors were found in the extremities (9/55, 16.3\%); chest/spine/ head and neck (27/55, 56.4\%); and, abdomen and pelvis (10/55, 27.3\%). IHC positivities for primary tumor specimens were CD99, 98\% (50/51); FLI-1, 92\% (24/26); vimentin, 85\% (11/13); and, CD56, 63\% (10/16). One specimen that was CD99-negative stained positive for vimentin and CD56.

We observed BM metastasis in seven patients (12.7\%, 7/55; Table 1). One patient was readily diagnosed as having BM metastasis of ESFT based on morphological findings (Fig. 1A \& B). IHC staining of CD56 and CD99 was performed for all seven specimens. Of these, three were CD56-/CD99+, and the others were CD56+/CD99+ (Fig. 1C \& D). We performed BM karyotyping for 42 patients, including three with BM metastasis (patients
Received: August 10,2020

Revision received: October 7, 2020

Accepted: March 5, 2021

Corresponding author: Chan-Jeoung Park, M.D., Ph.D.

Department of Laboratory Medicine, Asan Medical Center, University of Ulsan College of Medicine, 88 Olympic-ro 43-gil, Songpa-gu, Seoul 05505, Korea

Tel: +82-2-3010-4508, Fax: +82-2-478-0884

E-mail: cjpark@amc.seoul.kr 

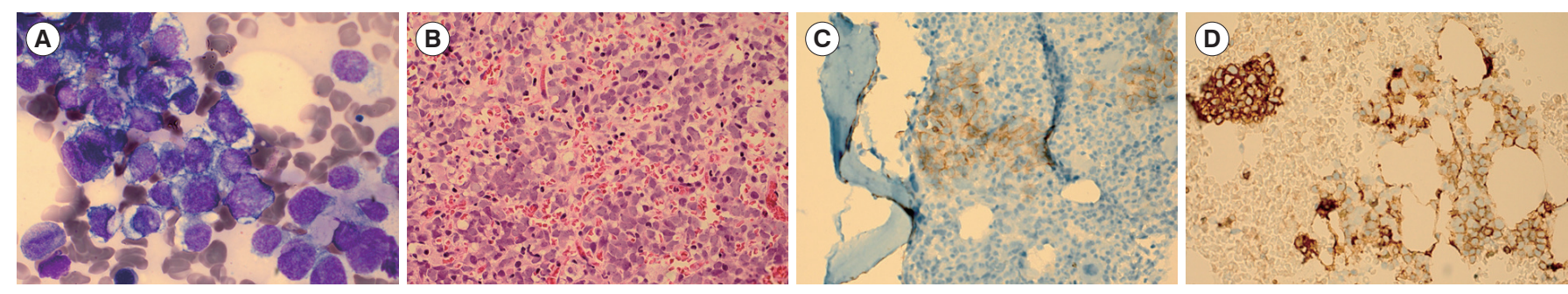

Fig. 1. Characteristic BM aspirate and biopsy findings of patients with BM metastasis of ESFT. (A) Small, round cells with bluish cytoplasm on BM aspirate smear (Wright stain, $\times 1,000)$. (B) Clustered, uniform, small, round cells in BM biopsy section (H\&E stain, $\times 400)$. (C) Clustered, neoplastic cells on BM biopsy section (CD56 IHC, $\times 400)$. (D) Clustered, neoplastic cells on BM clot section (CD99 IHC, $\times 400)$. Abbreviations: BM, bone marrow; IHC, immunohistochemistry; ESFT, Ewing sarcoma family tumor.

Table 1. Characteristics for seven patients with BM metastasis of Ewing sarcoma family tumor

\begin{tabular}{|c|c|c|c|c|c|c|c|}
\hline \multirow[b]{2}{*}{ Patient number } & \multirow[b]{2}{*}{ Sex/age } & \multirow{2}{*}{ Primary tumor site } & \multirow{2}{*}{$\begin{array}{l}\text { Metastatic finding, } \\
\text { bone scan/PET }\end{array}$} & \multirow{2}{*}{$\begin{array}{c}\text { BM site and BM } \\
\text { metastasis } \\
\text { (right/left, if bilateral) }\end{array}$} & \multirow{2}{*}{ BM karyotype } & \multicolumn{2}{|c|}{ BM IHC staining } \\
\hline & & & & & & CD56 & $\operatorname{CD99}$ \\
\hline 2 & $\mathrm{~F} / 17 \mathrm{yr}$ & Foot, left & $+/+$ & Unilateral, + & Not performed & + & + \\
\hline 3 & $\mathrm{~F} / 12 \mathrm{yr}$ & Abdominal mass & -/Not performed* & Bilateral, -l+ & Normal & + & + \\
\hline 6 & $\mathrm{M} / 6 \mathrm{yr}$ & Shoulder, right & +/Not performed & Bilateral, +/+ & Normal & + & + \\
\hline 7 & $\mathrm{~F} / 10 \mathrm{yr}$ & Ilium, right & $+/+$ & Bilateral, +/+ & Normal & - & + \\
\hline
\end{tabular}

*Multiple bone metastases on whole-body MRI.

Abbreviations: M, Male; F, Female; ESFT, Ewing sarcoma family tumor; BM, bone marrow; IHC, immunohistochemistry; PET, positron emission tomography; '+', positive; '-', negative; MRI, magnetic resonance imaging.

3, 6, and 7 in Table 1); all patients showed normal karyotypes.

Of the seven ESFT patients with BM metastasis, six had bone metastasis and one had lung metastasis. Bone scans did not show any distant bone metastatic findings in two BM metastasis cases; one case of multiple bone metastasis was revealed by whole-body magnetic resonance imaging (MRI), and the other case did not show any metastatic findings by positron emission tomography (PET).

This study focused on aiding accurate BM diagnosis for detecting BM metastasis of ESFT. Our findings suggest that IHC staining of CD56 and CD99 is useful for diagnosing BM metastasis of ESFT. In addition, imaging studies without BMAB may mislead the diagnosis of BM metastasis of ESFT.

In the present study, $12.7 \%$ of the ESFT patients had BM metastasis, while in other studies the BM metastasis rate varied between $2 \%$ and $18 \%$ [6-8]. Ethnic and institutional differences affecting patient characteristics, such as tumor burden, could explain this variability.

CD99 is a well-known diagnostic marker for ESFT [2]. However, one biopsy specimen of an ESFT primary site with no BM metastasis showed CD99 negativity, but CD56 positivity. Because CD56 positivity is reported in approximately $60 \%$ patients with ESFT [9], CD56 may serve as an additional diagnostic marker in BM evaluation of ESFT.

In our study, one BM metastasis case revealed no metastatic findings on whole-body PET/computed tomography and MRI. Although the limitation of BMAB for the detection of BM metastasis has been demonstrated, BMAB could reveal BM metastases in cases that did not show any metastatic findings in imaging studies [7, 8]. Furthermore, considering the very low risk of complications in BMAB [10] and its prognostic value in ESFT [3], it may be reasonable to perform BMAB for ESFT evaluation.

Our study has some limitations. First, the inclusion criterion was limited: patients who underwent BMAB. Second, recent technologies, such as next-generation sequencing that can detect circulating tumor DNA in the blood, could not be implemented in this study.

We conclude that BM study using IHC of CD56 and CD99 in ESFT is valuable for detecting metastasis. 


\section{ACKNOWLEDGEMENTS}

We thank all the patients and the medical staff for their contribution to this study. We would also like to thank Young Chan Lee, M.T. (Department of Laboratory Medicine, Asan Medical Center) for technical support in IHC staining and Editage (www. editage.co.kr) for English language editing.

\section{AUTHOR CONTRIBUTIONS}

Park CJ designed and directed the study. Cho YU, Jang S, and Seo EJ contributed to data interpretation. Park $\mathrm{K}$ analyzed the data and wrote the draft. Kim $\mathrm{H}$, Koh KN, and Im HJ treated patients, reviewed the manuscript, and provided critical feedback. All the authors approved the final manuscript.

\section{CONFLICTS OF INTEREST}

None declared.

\section{RESEARCH FUNDING}

None declared.

\section{ORCID}

Kuenyoul Park

Hyeri Kim

Kyung-Nam Koh

Ho Joon Im

Young-Uk Cho
Seongsoo Jang

Eul-Ju Seo

Chan-Jeoung Park

https://orcid.org/0000-0002-0045-1747

https://orcid.org/0000-0002-8247-3746

https://orcid.org/0000-0003-4396-8348

\section{REFERENCES}

1. Alava Ed, Lessnick SL, Stamenkovic I. Ewing sarcoma. In: WHO Classification of Tumours Editorial Board. WHO Classification of Tumours of Soft Tissue and Bone. 5th ed. Lyon: IARC Press, 2020:323-5.

2. Schaefer I-M and Hornick JL. Diagnostic immunohistochemistry for soft tissue and bone tumors: an update. Adv Anat Pathol 2018;25:400-12.

3. Thiel U, Wawer A, von Luettichau I, Bender HU, Blaeschke F, Grunewald TG, et al. Bone marrow involvement identifies a subgroup of advanced Ewing sarcoma patients with fatal outcome irrespective of therapy in contrast to curable patients with multiple bone metastases but unaffected marrow. Oncotarget 2016;7:70959-68.

4. Li X, Li W, Mo W, Yang Z. Acute lymphoblastic leukemia arising after treatment of Ewing sarcoma was misdiagnosed as bone marrow metastasis of Ewing sarcoma: a case report. Medicine (Baltimore) 2018;97: e9644.

5. Fernandez-Pol S, van de Rijn M, Natkunam Y, Charville GW. Immunohistochemistry for PAX7 is a useful confirmatory marker for Ewing sarcoma in decalcified bone marrow core biopsy specimens. Virchows Arch 2018;473:765-9.

6. Kasalak Ö, Glaudemans A, Overbosch J, Jutte PC, Kwee TC. Can FDG$\mathrm{PET} / \mathrm{CT}$ replace blind bone marrow biopsy of the posterior iliac crest in Ewing sarcoma? Skeletal Radiol 2018;47:363-7.

7. Cesari M, Righi A, Colangeli M, Gambarotti M, Spinnato P, Ferraro A, et al. Bone marrow biopsy in the initial staging of Ewing sarcoma: experience from a single institution. Pediatr Blood Cancer 2019;66:e27653.

8. Kopp LM, Hu C, Rozo B, White-Collins A, Huh WW, Yarborough A, et al. Utility of bone marrow aspiration and biopsy in initial staging of Ewing sarcoma. Pediatr Blood Cancer 2015;62:12-5.

9. Ash S, Luria D, Cohen IJ, Goshen Y, Toledano H, Issakov J, et al. Excellent prognosis in a subset of patients with Ewing sarcoma identified at diagnosis by CD56 using flow cytometry. Clin Cancer Res 2011;17:2900-7.

10. Bain BJ. Bone marrow biopsy morbidity: review of 2003. J Clin Patho 2005;58:406-8. 